\title{
Calidad de los registros clínicos de enfermería: Elaboración de un instrumento para su evaluación
}

\section{Nursing clinical registers quality: the generation of an assessment instrument}

Marisol Torres Santiago•

Rosa Amarilis Zárate Grajales.•

Reyna Matus Miranda...

\section{Resumen}

Introducción Los registros clínicos de enfermería conforman la evidencia escrita de los cuidados otorgados al paciente, son por excelencia un medio de comunicación y coordinación entre los profesionales de la salud, su realización correcta permite la continuidad de los cuidados y la seguridad del paciente. Objetivo: Construir y validar un instrumento para evaluar la calidad de los registros clínicos de enfermería. Metodología: Partiendo de la variable calidad de los registros clínicos de enfermería se realizó la búsqueda de información con respecto a la temática en diversas bases de datos como Pub Med, MEDLINE, Elsevier, Cochraner, se consultó la Ley General de Salud, la Norma Oficial Mexicana del Expediente Clínico (Nom-168-SSA-1998), el Código de Ética para Enfermeras y Enfermeros en México. Así como los estándares para la Certificación de Establecimientos de la Atención Médica del Consejo de Salubridad General. Con ello se consideró la normativa para el llenado del formato de los registros clínicos de enfermería, elaborada por la Subdirección de Enfermería perteneciente a una Unidad Médica de Tercer Nivel de Atención del sector público. Se construyó un instrumento que mide la calidad de los registros clínicos de enfermería (CARCE) en sus tres dimensiones estructura, continuidad de los cuidados y seguridad del paciente. Resultados: Para obtener la confiabilidad se utilizó el coeficiente Kuder-Richardson, alcanzando un índice de .86. Se aplicaron pruebas no paramétricas $U$ de Mann-Whinney y Kruskal Wallis para determinar el poder de discriminación entre las variables obteniendo diferencias estadísticamente signi-

-Licenciada en Enfermería. Alumna de la 6a Generación del Programa de Maestría en Enfermería de la Escuela Nacional de Enfermería y Obstetricia, Universidad Nacional Autónoma de México.

••Maestra en Investigación y Desarrollo de la Educación, Directora de Tesis, Escuela Nacional de Enfermería y Obstetricia, Universidad Nacional Autónoma de México.

...Maestra en Educación, Escuela Nacional de Enfermería y Obstetricia, Universidad Nacional Autónoma de México.

Correspondencia: marydamage19@yahoo.com.mx

Fecha de ReCibido: 3 Agosto de 2010.

FeCHA DE ENVIADO: 17 SEPTEMBRE DE 2010.

ACEPTADO: 5 NOVIEMBRE DE 2010. 


\section{Enfermería Universitaria}

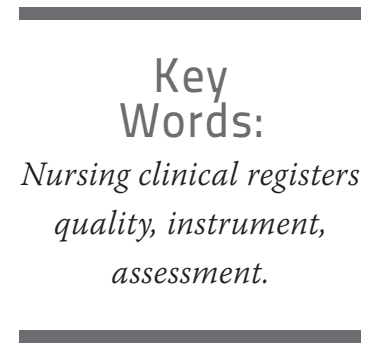

\section{INTRODUCCIÓN}

Las tendencias del mundo actual y la globalización en el campo de salud suponen nuevos desafíos para el profesional de enfermería en el logro de la calidad del cuidado, lo cual implica entre otras cosas el desarrollo de instrumentos que la evalúen de forma sistemática, con la finalidad de garantizar y ficativas p=0.0001. Discusión: Coincidiendo con el Modelo viPs un acrónimo para la documentación de enfermería que significa bienestar, integridad, prevención y seguridad vistos como los principales objetivos de los cuidados de enfermería y la perspectiva de Björvell C et al, quienes construyeron instrumentos para evaluar el grado en que los registros describen aspectos importantes del paciente en relación con la atención de enfermería. El instrumento CARCE aquí propuesto concuerda con los autores mencionados en virtud de que integra la continuidad del cuidado y la seguridad del paciente. Conclusiones: El instrumento reúne los elementos esenciales de confiabilidad y validez así como el poder de discriminación entre las variables intervinientes. Por lo anterior se propone como un instrumento para la evaluación objetiva de los registros clínicos de enfermería en instituciones de salud pública y privada.

\section{Abstract}

Introduction: nursing clinical registers represent written evidence of care given to patients, and a means of communication and coordination between health professionals; therefore, their correct constitution allows continuity in patient care security. Objective: Build and validate an instrument to assess nursing clinical registers quality. Methodology: we started searching in diverse databases including PubMed, MEDLINE, Elsevier, Cochraner, and also consulted the Health General Law, the Mexican Official Standard NOM-168-SSA-1998, the Ethical Code for Mexican Nurses, the standards of the General Health Council of the Medical Attention Sites Certification, and the Public Sector Third Level Attention Medical Units SubDirection. The instrument was named CARCE and it estimates three dimensions of care: structure, continuity, and patient security. Results: we calculated the KuderRichardson coefficient (.86) and the Mann-Whinney and Kruskal-Wallis coefficients to test variable-discrimination power and obtained statistically significant differences ( $p=.0001)$. Discussion: similar to the VIPS model, which tests well-being, integrity, prevention, and security seen as the principal nursing care objectives from the perspective of Bjorvell et al, the CARCE instrument was found to be useful to help integrate patient security and health care continuity. Conclusion: the instrument gathers the essential elements of validity and confidence as well as variable-discrimination power, and because of this, we propose it as an option to assess nursing clinical objectives both in public and in private institutions. optimizar los servicios prestados. Una de estas herramientas son los registros clínicos de enfermería ${ }^{1}$ los cuales deben estar orientados a documentar de forma permanente los cuidados en un marco éticolegal, además de ser considerados un indicador para la calidad del cuidado.
El Doctor Avedís Donabedian reconocido en el ámbito internacional como el máximo exponente en el estudio de la calidad de la atención sanitaria, considera que ésta es la atención que se espera pueda proporcionar al paciente el máximo y más completo bienestar en todo momento, logrando 
los mayores beneficios posibles. A su vez existen atributos de la atención que se convierten en determinantes mucho más poderosos de su calidad como son la continuidad y la coordinación de los cuidados. Un rasgo fundamental del primer atributo es la preservación de la información acerca de los hallazgos entre el equipo de salud. ${ }^{2}$ La continuidad y la coordinación son mejores si la responsabilidad está centralizada en las manos de profesionales y, en el caso que más de uno de éstos estén involucrados deben ser realizadas en forma ordenada en particular si la responsabilidad se transfiere o se comparte. $^{3}$

En este orden de ideas, la Joint Commission conceptualiza como objetivo de la continuidad de los cuidados; el definir, dar forma y ordenar los procesos y actividades para potenciar al máximo la coordinación dentro de la atención asistencial. ${ }^{4}$ En este mismo tenor Eggland y Heinemann señalan la continuidad como la transferencia sin obstáculos del cuidado de una persona entre proveedores destacando como puntos importantes la comunicación de la información y la responsabilidad en el cuidado. ${ }^{5}$

Lo anterior resulta trascendental como lo observado por Zimmerman et al, quienes mostraron a través de un estudio multicéntrico cómo a través de una comunicación efectiva y elevados niveles de coordinación alrededor de una cultura centrada en el paciente, se alcanzaban cifras de estancia y mortalidad inferiores. Concluyendo en este estudio, que la comunicación entre unidades y equipos de atención en el momento del cambio de turno podría no incluir toda la in- formación esencial, o bien podría darse una interpretación incorrecta de ésta; estas brechas en la comunicación pueden provocar graves interrupciones en la continuidad de la atención, un tratamiento inadecuado e incluso daño potencial para el paciente. ${ }^{6}$ De esta manera entre 1995 y 2006 la Organización Mundial de la Salud (OMS) refiere que la falta de comunicación durante el cambio de turno fue la causa principal de los eventos adversos denunciados ante la Joint Commission siendo el factor causal más común de reclamos surgidos de transferencias ante las agencias de seguros por mala praxis en EE.UU. Incluso que de los 25000 a 30000 eventos adversos todos ellos evitables que condujeron a una discapacidad de los pacientes en Australia, el 11\% se debió a problemas de comunicación, en contraste con el 6\% debido a niveles de competencia inadecuados de los facultativos. ${ }^{7}$

Otro aspecto íntimamente relacionado con la calidad es el referente a la seguridad del paciente, la cual es conceptualizada por el Instituto de Medicina de la Academia Nacional de Ciencias de los Estados Unidos como el conjunto de

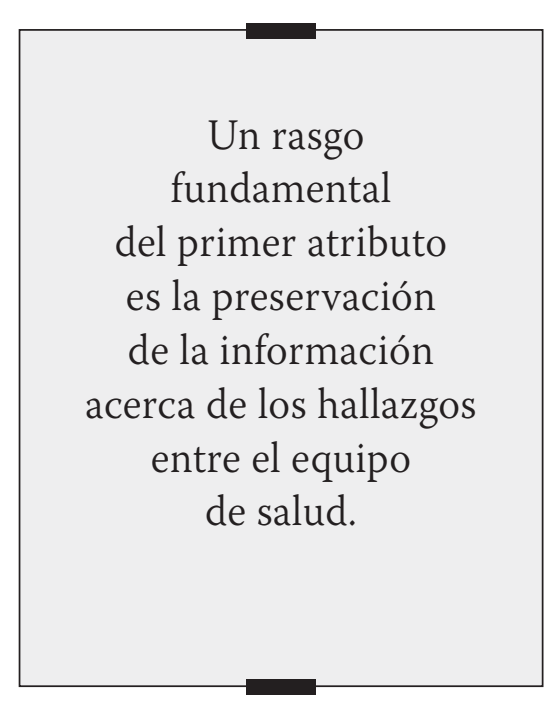

elementos y metodologías basadas en evidencia científicamente probada, para minimizar el riesgo de sufrir un evento adverso en el proceso de atención de salud o mitigar sus consecuencias. ${ }^{8}$ Tomando en cuenta lo anterior, la seguridad forma parte de la caracterización de calidad de un servicio de salud en conjunto con la oportunidad, la accesibilidad, la pertinencia y la continuidad de los cuidados.

De acuerdo con el Plan Nacional de Desarrollo 2007-2012 (PND) de nuestro país, uno de los objetivos centrales de la política pública en salud es brindar servicios eficientes, con calidad y seguridad para el paciente. Por lo que se establece como instrumento operativo el Sistema Integral de Calidad en Salud (SICALIDAD), encargado de elevar la calidad de estos servicios con un enfoque en la mejora de la calidad técnica y la seguridad del paciente, la calidad percibida y la calidad en la gestión de los servicios de salud. Desde una perspectiva institucional, SICALIDAD se convierte en la estrategia nacional que sitúa la calidad en la agenda permanente del Sistema Nacional de Salud.

En los proyectos de SICALIDAD se puede localizar dentro de la dimensión de calidad técnica y seguridad del paciente, el expediente clínico integrado y de calidad; entre las acciones principales se destaca establecer un diagnóstico de los aspectos críticos que han afectado el cumplimiento de la Norma Oficial Mexicana del expediente clínico (NOM 168-SSA-1998) la cual representa el instrumento para la regulación de éste y orienta al desarrollo de una cultura de la calidad. ${ }^{9}$ Esta Norma en su apartado 9.1 hace referencia a la hoja de enfermería, 


\section{Enfermería Universitaria}

señalando las consideraciones mínimas que debe contener; habitus exterior, gráfica de signos vitales, ministración de medicamentos con la fecha, hora, cantidad y vía, procedimientos realizados y observaciones de enfermería. ${ }^{10}$

Tomando en cuenta lo anterior la Comisión Permanente de Enfermería que es un grupo colegiado conformado por representantes institucionales del sistema de salud mexicano tanto del sector público y privado así como del sector educativo y grupos profesionales de enfermería unidos en beneficio de la calidad, de la formación y el desarrollo profesional del personal de enfermería, ${ }^{11}$ durante la 1era Jornada Técnica celebrada del 1 al 2 de julio 2008, concluye que documentar los cuidados de enfermería resulta imprescindible en una estrategia de calidad. ${ }^{12}$

En este orden de ideas, los expertos señalan que la evaluación es una de las mejores tácticas para garantizar que los servicios de enfermería sean de alta calidad. Por lo que se debe comparar la situación real del sistema de atención con los objetivos propuestos por la gerencia, lo anterior implica el monitoreo y seguimiento de dichos servicios con base en estándares e indicadores de estructura, proceso y resultado para medir periódicamente la ejecución de actividades. ${ }^{13}$

Considerando lo anterior existen una gama de estudios e instrumentos creados por enfermeras para evaluar la calidad del cuidado: como el estudio Nursing Audit realizado con el objetivo de evidenciar el cumplimiento de ciertas funciones de evaluación de la calidad a través de la documentación de cuidados de los pacientes dados de alta. Se concluye que la evaluación de la documentación ha de hacerse, ya que muestra una parte importante de la calidad de los cuidados que ha recibido el paciente. ${ }^{14}$

Por su parte la Joint Commission on Acreditation of Health Care Organization ha establecido los criterios para evaluar el registro de la atención y seguimiento del paciente, por lo que exige la documentación en el contexto del Proceso de atención de enfermería, en particular, la educación dirigida al paciente y/o familiar y la producción del plan de alta si así lo requiriera. ${ }^{15}$

Bajo esta perspectiva, Björvell C et al, construyeron un instrumento para evaluar el grado en que los registros describen aspectos importantes del paciente en relación con la atención de enfermería. En este estudio concluyen que la auditoría de los registros de los pacientes se puede hacer por varias razones, lo más común como parte de un proceso continuo de mejora de la calidad de los registros y para cambiar ciertos comportamientos de los profesionales de la salud. ${ }^{16}$.

De esta manera general se concluye que la información contenida en los registros clínicos de enfermería debe ser completa, concisa, apropiada y total sobre el cuidado del paciente lo que significa que las observaciones deben ser actuales e inmediatas a los cuidados para evitar la omisión o duplicación de la información, a su vez ésta se debe transmitir en un orden lógico. ${ }^{17}$

Siguiendo algunas de las pautas con relación a la elaboración de los registros clínicos de enfermería para mantener la calidad de los mismos las anotaciones deben ser legibles, utilizar tinta permanente, incluyendo la fecha y la hora de cada una de ellas, la ortografía es indispensable para la exactitud de los registros, utilizar abreviaturas y símbolos universales, no solo por cuestiones legales sino también por seguridad del paciente. ${ }^{18}$

Por tal motivo y de acuerdo con Owen K. la seguridad del paciente es primordial y el mantenimiento de los registros clínicos de enfermería debe seguir siendo un aspecto fundamental en la atención de enfermería debiendo ser un objeto de auditoría como medida de control de calidad. ${ }^{19}$

Tomando en cuenta todo lo antes señalado, en este documento se muestra el proceso de elaboración y validación de un instrumento de medición de la calidad de los registros clínicos de enfermería, señalando que ésta es la primera etapa de un proyecto de investigación más amplio.

\section{Material y Método}

Inicialmente llevó a cabo la búsqueda de información con respecto a la temática en diversas bases de datos como Pub Med, MEdline, Elsevier, Cochrane entre otras; inmerso en tal hecho y como eje central se consideró la normativa que regula la responsabilidad legal y ética para los profesionales de la salud en México, es decir, se consultó la Ley General de Salud, la Norma Oficial Mexicana Del Expediente Clínico (NOM-168-SSA-1998), el Código de Ética para Enfermeras y Enfermeros en México. Así como los estándares para la Certificación de Establecimientos de la Atención Médica del Consejo de Salubridad General en especial lo concerniente con la auditoría de los registros clínicos de enfermería en el Expediente Clínico; por último se consideró la nor- 
mativa para el llenado del formato de los registros clínicos de enfermería, elaborada por la Subdirección de Enfermería perteneciente a una Unidad Médica de Tercer Nivel de Atención del sector público. ${ }^{20}$

Posteriormente se conceptualizó la variable calidad de los registros clínicos de enfermería como la evidencia escrita de los actos del cuidado que realiza el profesional de enfermería, que si no cumple con los estándares propuestos repercutirá en la continuidad del cuidado y la seguridad del paciente.

Con la premisa antes señalada inició la primera fase de la construcción del instrumento; ésta consistió en realizar la descripción del procedimiento de los registros clínicos de enfermería con base a la normativa revisada.

Se utilizaron herramientas administrativas para la descripción gráfica del procedimiento por medio de un diagrama de flujo a fin de representar las actividades de forma secuencial durante la elaboración de los registros clínicos de enfermería e identificar las áreas críticas a evaluar, también se diseñó un diagrama PEPSU (Proveedores, Entradas, Procesos, Salidas, Usuarios) en donde se sintetizó el proceso y se establecieron los elementos indispensables para su desarrollo como las entradas, proveedor, los procesos intermedios, las salidas y el usuario para identificar los límites de responsabilidad.

Con lo anterior se desarrollaron 20 enunciados considerados como puntos críticos para ser evaluados, éstos contemplaron la primera fase del instrumento. Desde ese momento se decidió que la escala de respuestas sería dicotómica en

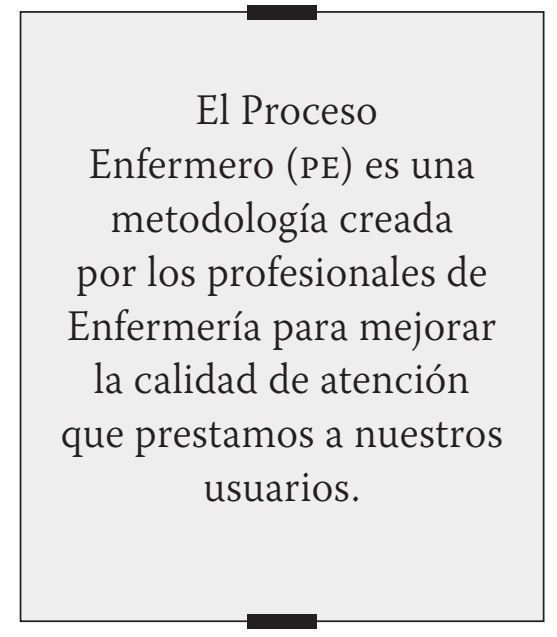

donde "Presente" tendría un código de valor de 1 sí se encontraba el registro clínico y "Ausente" con un valor de 0 .

En la segunda fase se determinó que el constructo calidad de los registros clínicos de enfermería estaría conformado por tres dimensiones: estructura, continuidad del cuidado y seguridad del paciente. (Cuadro 1) De esta manera se concluyó la segunda fase de la construcción del instrumento conformado por un total de 27 ítems.

Para alcanzar la validez de contenido, el instrumento, se sometió a una ronda de siete expertos, considerados por su conocimiento, experiencia en el manejo y dominio de la temática en instituciones del sector salud y educativo tanto público como privado.

A éstos se les dio a conocer el nombre del proyecto, objetivo y las partes en que estaba constituido el instrumento, es decir, las variables intervinientes: servicio, turno, edad, sexo, antigüedad laboral y nivel académico del personal de enfermería y los enunciados que evalúan el grado de calidad de los registros clínicos de enfermería a través de la estructura, continuidad del cuidado y la seguridad del paciente; los expertos evaluaron la funcionalidad de cada uno de los enunciados y se les solicitó que emitieran un comentario final.

De acuerdo a la opinión de cada uno de ellos, se reestructuraron algunos enunciados en cuanto a la semántica, el orden de los ítems se modificó de acuerdo a las etapas del Proceso Atención de Enfermería (PAE) y se decidió incorporar cinco ítems considerados pertinentes para la finalidad del estudio.

Lo anterior, originó una tercera fase y con ella un instrumento para evaluar la calidad de los registros clínicos de enfermería (CARCE) conformado por 32 ítems, incorporándose un apartado para observaciones en donde se incluyen datos convenientes para el análisis de resultados.

Previa autorización de la Subdirección de Enfermería y del Comité de Ética de una Unidad Médica de Tercer Nivel de Atención del D.F. del 3 al 15 de marzo del 2010 se efectuó la prueba piloto donde fue aplicado el instrumento. Se determinó una muestra por conveniencia de 60 notas de enfermería.

La unidad de análisis fueron los registros clínicos de enfermería contenidos en los expedientes clínicos, éstos se eligieron entre aquellos que cumplían con los siguientes criterios de inclusión: a) De pacientes egresados por mejoría o alta voluntaria de 3 servicios: Cirugía Plástica (30), Reumatología (15) y Endocrinología (15), b) registros clínicos elaborados por personal de enfermería adscrito y de los turnos matutino, vespertino y nocturno, c) se consideraron todos los niveles académicos del personal: Auxiliar de enfermería, Enfermera General, Postécnico, Licenciatura y Posgrado. 


\section{Cuadro 1. Constructo Calidad de los Registros clínicos de Enfermería}

\begin{tabular}{|c|c|c|c|}
\hline Dimensiones & Definición & $\begin{array}{l}\text { No. } \\
\text { İtems }\end{array}$ & Fase \\
\hline Estructura & $\begin{array}{l}\text { Se refiere a aspectos "de forma" del documento, es decir, los } \\
\text { registros clínicos deben ser estructurados por medio de un len- } \\
\text { guaje técnico-científico, comprensible a todos los miembros del } \\
\text { equipo de salud. Para lo cual se deben excluir faltas de ortogra- } \\
\text { fía, legibles, utilizar abreviaturas y símbolos universales. }\end{array}$ & 9 & 1 \\
\hline $\begin{array}{l}\text { Continuidad } \\
\text { del cuidado }\end{array}$ & $\begin{array}{l}\text { Caracterizándose por la no interrupción del cuidado y su man- } \\
\text { tenimiento a través del registro, de acuerdo a las etapas del } \\
\text { Proceso de Atención de enfermería: valoración, diagnósticos de } \\
\text { enfermería, intervenciones y evaluación. } .^{21}\end{array}$ & $\begin{array}{l}10 \\
9 \\
10\end{array}$ & 1 \\
\hline $\begin{array}{l}\text { Seguridad } \\
\text { del paciente }\end{array}$ & $\begin{array}{l}\text { Reducción de riesgos innecesarios derivados de la atención de } \\
\text { salud por medio de la información escrita sobre las medidas de } \\
\text { seguridad en prevención de caídas, errores en medicación, úlce- } \\
\text { ras por presión, alergias, etc. }\end{array}$ & $\begin{array}{r}9 \\
10\end{array}$ & 2 \\
\hline
\end{tabular}

\section{Cuadro 2. Medianas globales del puntaje alcanzado por la variable antigüedad laboral}

\section{Antigüedad laboral}

1 a 13 años

$\mathrm{n}=41$

14. y > años

$\mathrm{n}=19$

\section{Medianas globales}

21

(1 a 12)

28

(1 a 18)

Prueba U de Mann-Whiney. " $\mathrm{p}=0.0001$ 


\section{Cuadro 3. Medianas globales de la Calidad de los Registros Clínicos de Enfermería por dimensiones y servicios}

\begin{tabular}{|c|c|c|c|c|}
\hline Servicio & $\begin{array}{l}\text { Dimensiones } \\
\text { Estructura }\end{array}$ & $\begin{array}{l}\text { Continuidad } \\
\text { del cuidado }\end{array}$ & $\begin{array}{l}\text { Seguridad } \\
\text { del paciente }\end{array}$ & ${ }^{*} p$ \\
\hline $\begin{array}{l}\text { Cirugía Plástica } \\
\mathrm{n}=30\end{array}$ & $\begin{array}{c}7.5 \\
(5 \text { a } 10)\end{array}$ & $\begin{array}{c}6.0 \\
(3 \text { a } 8)\end{array}$ & $\begin{array}{c}4.0 \\
(2 \mathrm{a} 6)\end{array}$ & .001 \\
\hline $\begin{array}{l}\text { Reumatología } \\
\mathrm{n}=15\end{array}$ & $\begin{array}{c}9.0 \\
(6 \text { a } 11)\end{array}$ & $\begin{array}{c}4.0 \\
(3 \mathrm{a} 6)\end{array}$ & $\begin{array}{c}4.0 \\
(3 \mathrm{a} 6)\end{array}$ & .001 \\
\hline $\begin{array}{l}\text { Endocrinología } \\
\mathrm{n}=15\end{array}$ & $\begin{array}{c}8.0 \\
(3 \mathrm{a} 10)\end{array}$ & $\begin{array}{c}5.0 \\
(3 \text { a } 7)\end{array}$ & $\begin{array}{c}4.0 \\
(3 \mathrm{a} 6)\end{array}$ & .001 \\
\hline
\end{tabular}

${ }^{*}$ p Prueba Kruskal-Wallis

Con respecto al plan de análisis estadístico se elaboró una base de datos y se apoyó con el paquete estadístico Statistical Package for the Social Sciences (SPSS) versión 18.0. Para comparar las medianas globales obtenidas de los grupos incluidos en la prueba piloto.

\section{Resultados}

Con el fin de estimar la confiabilidad del instrumento, se empleó el coeficiente Kuder-Richardson en los instrumentos aplicados en la prueba piloto, obteniéndose un índice de $r=.86$. Para determinar el poder de discriminación del instrumento entre las variables intervinientes como la antigüedad laboral se utilizó la prueba U de Mann-Whiney donde se obtuvo una $\mathrm{U}=62.92, \mathrm{p}=<0.05$. (Cuadro 2) La prueba Kruskal-Wallis se utilizó con la variable servicio donde $\mathrm{X}^{2}=182.50, \mathrm{gl}=2, \mathrm{p}=<0,05 \mathrm{y}$ entre las dimensiones del constructo calidad de los registros clínicos de enfermería donde $\mathrm{X}^{2}=20.83, \mathrm{gl}=2$, $\mathrm{p}=0.001$; encontrándose diferencias estadísticamente significativas. (Cuadro 3)

\section{Discusión}

A medida que aumenta la complejidad de la asistencia sanitaria, y los límites entre los profesionales de la salud, los sectores se tornan más complicados por lo que la capacidad de comunicación efectiva sobre la atención de los pacientes se vuelve más importante que nunca. El desarrollo de registros de enfermería se centra en su uso como vehículos para el almacenamiento e intercambio de información; los beneficios esperados de un registro consistente y dirigido de información acerca de la atención al paciente son claros e incluyen la seguridad

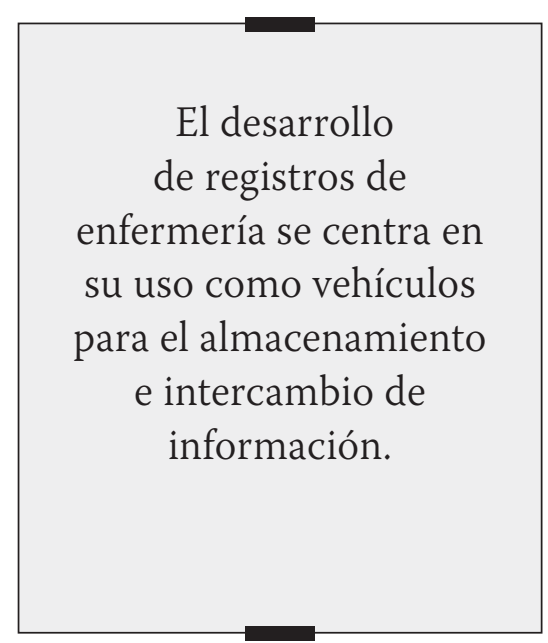

del paciente y la continuidad de los cuidados. ${ }^{1}$

Ante tal premisa, en diversos países, los profesionales de enfermería se han dado a la tarea de construir instrumentos para evaluar la calidad de los registros clínicos como Rosendal DM et al, quienes evaluaron el Modelo VIPS un acrónimo para la documentación de enfermería que significa bienestar, integridad, prevención y seguridad vistos como los principales objetivos de los cuidados de enfermería.22 Bajo esta perspectiva, Björvell $\mathrm{C}$ et al, construyeron un instrumento para evaluar el grado en que los registros describen aspectos importantes del paciente en relación con la atención de enfermería. La documentación de enfermería debe ser diseñada para que contribuya a garantizar la seguridad del paciente y proporcionar las bases para la evaluación continua y la revisión de las intervenciones de enfermería.

Se coincide que el instrumento de evaluación de la calidad de los registros clínicos de enfermería (CARCE) aquí presentado inclu- 
ye el Proceso de Atención de enfermería como lenguaje universal para describir y efectuar los registros clínicos y reflejar las funciones independientes, interdependientes y dependientes del profesional de enfermería y su contribución a la calidad y seguridad de la atención a la salud

En la etapa de valoración, se coincidió con Miró BM et al, quienes tomaron en consideración la valoración de alergias, del dolor, la presencia y aspecto de los accesos vasculares, la presencia de drenajes, heridas quirúrgicas, barreras idiomáticas, información sobre el soporte religioso, la valoración de úlceras por presión así como el riesgo de caídas, ${ }^{23}$ el instrumento considera también las medidas de prevención aplicadas ante la vulnerabilidad del paciente.

Otro elemento incorporado en el instrumento CARCE es el informe de alta que permite la continuidad de los cuidados en el hogar y la comprensión de la terapéutica para el paciente y la familia y, por lo tanto refleja la calidad de la asistencia, como lo describe Simón GMJ et al. ${ }^{24}$ Aspectos fundamentales para la elaboración de los registros clínicos de enfermería se identifican como es la letra legible, el uso de terminología científica, la utilización de abreviaturas y unidades de medición universal e institucionalmente aceptadas como hace referencia Fay $\mathrm{YR}^{25}$ y que para fines de la calidad de los registros clínicos dichos elementos constituyen la dimensión de estructura en el instrumento CARCE.

Por lo tanto este instrumento permite también evaluar el cumplimiento de la Norma Oficial Mexicana 168 Del Expediente Clí- nico (NOM 168); ya que ante cualquier demanda por negligencia profesional se acude a éste para analizar la relación escrita de los hechos. ${ }^{26,27}$ También se contemplan los estándares para la Certificación de Establecimientos de Atención Médica del Consejo de Salubridad General en México con respecto a la hoja de enfermería ${ }^{28}$ actualmente el profesional de enfermería es activo y proactivo en el proceso de certificación y acreditación en las instituciones de salud.

Finalmente a través de la prueba piloto se pudo estimar las fortalezas metodológicas como la confiabilidad, validez y el poder de discriminación entre las variables intervinientes del instrumento de medición CARCE. El cual se puede afirmar reúne los puntos críticos que debe contemplar la documentación de enfermería con estándares de calidad y seguridad para el paciente y por lo tanto puede ser utilizado en instituciones de salud privadas como públicas.

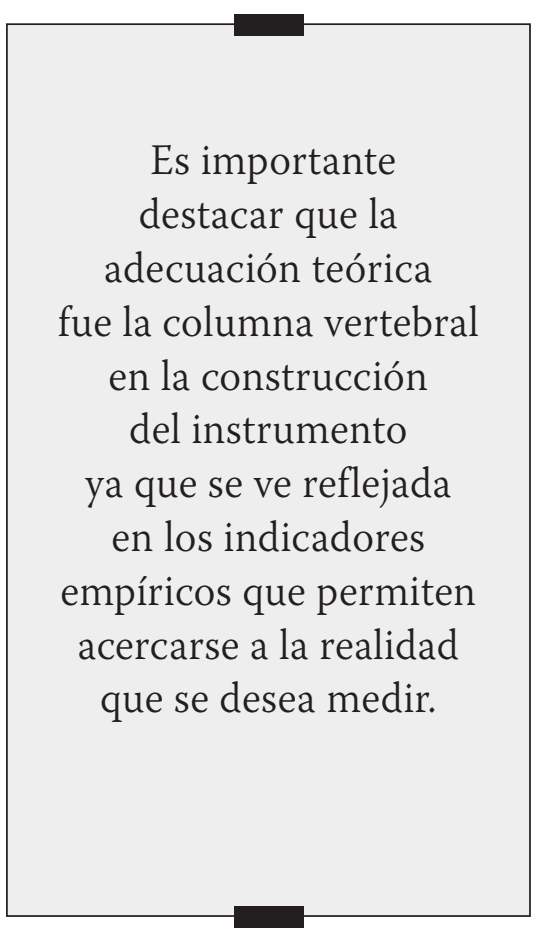

En un segundo momento se darán a conocer los resultados obtenidos sobre la calidad de los registros clínicos de enfermería en una Unidad Médica de Tercer Nivel de Atención del D.F, México. Es importante destacar que la adecuación teórica fue la columna vertebral en la construcción del instrumento ya que se ve reflejada en los indicadores empíricos que permiten acercarse a la realidad que se desea medir.

\section{Referencias}

1 Currell R, Urquhart C. Sistema de registro de enfermería: efectos sobre la práctica de la enfermería y resultados en la asistencia sanitaria (Revisión Cochrane traducida), En: La Biblioteca Cochrane Plus, 2008 Número 2, Oxford: Update Software Ltd.

2 Donabedian A. La calidad de la atención médica. DF, México: La Prensa Médica Mexicana; 1984.

3 Donabedian A. Continuidad y cambio en la búsqueda de la calidad. Rev Calidad de Atención a la Salud 1995;2(3):8-17.

4 Joint Commssion on Acreditation of Healthcare Organizations: Características de los indicadores clínicos. Control de Calidad Asistencial 1991; 6:65-74.

5 Eggland ET, Heinemann DS. Nursing documentation: charting, recording and reporting. Philadelphia: Lippincott, 1994.

6 Zimmerman JE, Shortell SM, Rousseau DM, Duffy J, G illies RR, Knaus WA, et al. Improving intensive care: observations based on organizational case studies in nine intensive care units: a prospective, multicenter study. Crit Care Med 1993; 21:1443-51. 
7 Citado por World Health Organization. World Alliance for patient safety. Ginebra, Suiza, 2007. Disponible en: http://www.who.int/ patientsafety/en/ (Consultado 16 Junio de 2009)

8 Kohn LT, Corrigan JM, Donaldson MS. To err is human: building a safer health system. Washington: National Academic Press, 2000.

9 Subsecretaría de Innovación y Calidad. Programa de Acción específica 2007-20012.Sistema Integral de Calidad en Salud SICALIDAD, México, p 11-32.

10 Norma Oficial Mexicana Del expediente clínico NOM-168SSA1-1998. Disponible en: http:// www.salud.gob.mx/unidades/ cdi/nom/168ssa18.html (Consultado el 23 Abril de 2009)

11 Jiménez AJ. En el camino hacia la mejora de la calidad, estandarización y fundamentación de los cuidados de enfermería. Rev CONAMED 2008;13( 2):3-5.

12 Comisión Interinstitucional de Enfermería. $1^{a}$ Jornada Técnica Plan de cuidados de enfermería. Disponible en: www.salud.gob.mx/unidades/cie/cms_cpe (Consultado el 20 Junio de 2009)

13 Ortega VC, Suárez VG. Manual de Evaluación de la Calidad del Servicio de Enfermería, 2a ed. D.F, México: Panamericana; 2009.
14 Peya GM. Un repaso de la evolución de los estudios de calidad de los cuidados de enfermería. Nursing. España, 2004;22(9):56-65.

15 Citado por Potter P. Fundamentos de Enfermería. $5^{a}$ ed. España: Mosby; 2003.Vol. I. p 514-8.

16 Bjorvell C, Thorell I, Wredling R. Development of an audit instrument for nursing care plans in the patient record. Quality in Health Care 2000;9(6):6-13.

17 Potter P, Fundamentos de Enfermería. 5a ed. España: Mosby; 2003. Vol. I. p 513-6.

18 Kozier B. Fundamentos de Enfermería. D.F, México: Mc Graw Hill; 1994.

19 Owen K. Documentation in nursing practice, Art and science community nursing 2005; 19(32):48-9.

20 Hospital General de México OD. Subdirección de Enfermería. Instructivo de manejo y llenado del formato registros de enfermería. D.F, México; 1997.

21 Carpenito KJ. Planes de cuidados y documentación clínica en enfermería .4 ed. Colombia: Mc Graw Hill Interamericana; 2005.

22 Rosendal DM, Geissler NB, Landberger G, Lippert E, Egerod I. Nursing documentation audit-the effect of o VIPS implementation programme in Denmark. Journal compilation. Denmark. 2006: 526-34.
23 Miró BM et al. Valoración al ingreso del paciente crítico. Un indicador de calidad asistencial. Enfermería Intensiva 2000; 11(2):51-8.

24 Simón GMJ, et al. Efectividad de un programa de mejora para la calidad de los registros enfermeros en ginecología y obstetricia. Enferm Clin 2006;16(6):300-5.

25 Fay YR. Registros de enfermería: cuidados de calidad. Nursing 2003; 21(3):23-27.

26 Austin S. Aspects of nursing documentation. Nursing 2007; 25(1): 20-6

27 Méndez CG, et al. La trascendencia que tienen los registros de enfermería en el expediente clínico. Revista Mexicana de Enfermería Cardiológica 2001; (1-4):11-7.

28 Consejo de Salubridad General. Comisión para la Certificación de Establecimientos de Atención Médica. Estándares para la Certificación de Hospitales. Manual del Proceso de Certificación. DF, México. 2009. 